\title{
Establishing private communications in open systems using multicapabilities
}

\begin{abstract}
Private and secure communications can be vital for certain applications, for example secure bidding, e-commerce, and online bank transactions. However, it is impossible to carry out private conversations in the open tuple-space model, despite it being a popular coordination model for distributed, heterogeneous systems, where agents can communicate via a common data space without having to know each other's identity, or having to arrange for a definite rendezvous. In this paper, we present an algorithm for establishing private communications in tuple-space systems using capabilities. In view of the fact that open systems need to be scalable, capabilities may provide a finer control to the loose control coordination of tuplespace systems whilst maintaining their flexibility. As capabilities can only refer to uniquely identifiable objects, we have introduced the concept of multicapabilitiesð capabilities for groups of unnamed objectsð to be applied to nameless tuples.
\end{abstract}

Keyword: Private communication; Tuple-space; Multicapabilities; LINDA; Open systems 